
\title{
$\begin{array}{ll}\text { Research Square } & \text { They should not be considered conclusive, used to inform clinical practice, } \\ \text { or referenced by the media as validated information. }\end{array}$
}

\section{Efficacy and Safety of Immunotherapy Compared With Chemotherapy in The Second-Line Treatment of Advanced Esophageal Cancer: A Meta-Analysis and System Review}

\section{Kang-hao Zhu}

Taizhou Hospital of Zhejiang Province

\section{Cong-cong $\mathrm{Xu}$}

Taizhou Hospital of Zhejiang Province

\section{Dong Chen}

Taizhou Hospital of Zhejiang Province

\section{Zi-xian Jin}

Taizhou Hospital of Zhejiang Province

\section{Si-jia Ren}

Taizhou Hospital of Zhejiang Province

Jian-fei Shen ( $\sim$ jianfei051@163.com )

Wenzhou Medical University

\section{Bao-fu Chen}

Taizhou Hospital of Zhejiang Province

\section{Research}

Keywords: esophageal cancer, meta-analysis, immunotherapy

Posted Date: November 16th, 2021

DOI: https://doi.org/10.21203/rs.3.rs-1043624/v1

License: (c) (i) This work is licensed under a Creative Commons Attribution 4.0 International License. Read Full License 


\section{Abstract}

Background: Currently, surgical resection, radiotherapy, and chemotherapy are most often utilized in clinical practice, however, they are associated with limited survival benefits. Immunotherapy is currently widely used as the second-line treatment of advanced esophageal cancer, but its effectiveness and safety compared to chemotherapy for advanced esophageal cancer have yet to be proven.

Methods: Original articles describing the safety and effectiveness of immunotherapy for advanced esophageal cancer published before February 2021 were retrieved from PubMed, Embase and Cochrane libraries. Hazard ratio (HR) and 95\% confidence interval $(\mathrm{Cl})$ were calculated and heterogeneity analysis was performed.

Results: A total of four studies were included, involving 2087 patients with esophageal cancer. They were included in the meta-analysis and compared immunotherapy and chemotherapy in the treatment of esophageal cancer. Overall survival, progression-free survival, treatment-related adverse events, and objective remission rate were used to evaluate the safety and effectiveness of immunotherapy. Our analysis showed that compared with chemotherapy, immunotherapy gave a significant survival advantage in patients with esophageal cancer ((HR 0.79, 95\% Cl 0.71-0.88), and fewer treatmentrelated adverse events at grade 3 or above. Among them, immunotherapy showed high safety ( $\mathrm{HR} 0.38,95 \% \mathrm{Cl} 0.28$ $0.51)$, but there was no significant difference in progression-free survival between the two groups $(\mathrm{HR} 1.01,95 \% \mathrm{Cl} 0.79$ 1.30), The objective response rate showed that immunotherapy has an advantage over chemotherapy $(\mathrm{HR} 1.67,95 \% \mathrm{Cl}$ 0.91-3.16).

Conclusion: For patients with advanced esophageal cancer, immunotherapy is more effective and safer than chemotherapy as the second-line treatment.

\section{Introduction}

Esophageal cancer ranks seventh in terms of incidence $(604,000$ new cases) and sixth in mortality overall $(544,000$ deaths), the latter signifying that esophageal cancer is responsible for one in every 18 cancer deaths in 2020.

Esophageal cancer mainly occurs in East Asia, especially in China, which has the greatest burden [1]. Esophageal cancer is mainly divided into two subtypes, esophageal adenocarcinoma and esophageal squamous cell carcinoma, and $90 \%$ of esophageal cancers in Asia are esophageal squamous cell carcinoma. At present, surgical resection, radiotherapy and chemotherapy are the main clinical treatment methods for esophageal cancer. However, due to the limited efficacy and serious adverse reactions of conventional treatments, the results are still unsatisfactory [2, 3]. Surgery is not effective for advanced or metastatic esophagus, and chemotherapy, such as taxanes, cisplatin, and fluorouracil, is usually used as the first-line treatment in these cases. However, there are few second-line treatment options for patients with advanced or metastatic esophageal squamous cell carcinoma who have progressed or cannot receive first-line standard chemotherapy. Moreover, the programmed death ligand-1 (PD-L1) inhibitor pembrolizumab has been approved as a highly efficient drug for patients with PD-L1-positive or advanced esophageal squamous cell carcinoma (ESCC). These novel drugs can be used alone or in combination with other treatment strategies to further improve the treatment efficacy and prognosis of cancer patients [4-6]. And nivolumab, as an anti-PD-1 antibody, has been accepted globally as a standard second-line treatment option. There are many other PD-L1 inhibitors of the same type as pembrolizumab, but their safety needs to be confirmed.

As there is only meta-analysis [7] for resectable esophageal cancer, we use overall survival rate, adverse event rate, and progression-free survival and other indicators to compare and analyze randomized controlled trials of immunotherapy and chemotherapy for locally advanced or metastatic esophageal cancer. The purpose of our meta-analysis is to verify the efficacy and safety of immunotherapy compared to chemotherapy. 


\section{Materials And Methods}

This meta-analysis tries to follow the Preferred Reporting Items for Systematic Reviews and Meta-Analyses (PRISMA) guidelines [8]. At present, there is no analysis as outlined above.

\section{Search strategy and study selection}

PubMed, Embase and Cochrane libraries were used to conduct a comprehensive English search for articles on immunotherapy of esophageal cancer reported before January 1, 2021. At the same time, we also searched for the latest data from clinical trials of immunotherapy for advanced esophageal cancer that have not been published as of January 1, 2021 at American Society of Clinical Oncology (ASCO), European Society of Medical Oncology (ESMO) and other international tumor conferences. We used medical keywords to search for esophageal cancer (including esophageal squamous cell carcinoma, esophageal adenocarcinoma), PD-L1, PD-1, CTLA-4, chemotherapy and immunotherapy.

The clinical trials that met the following criteria were included in this study: 1 . The publication reported verifiable advanced unresectable esophageal cancer; 2. Immune checkpoint inhibitors (ICls)were currently used in clinical practice or in registered clinical trials; 3 . The report included a complete plan, patient data and at least one key data such as overall survival rate, progression-free survival rate, incidence of adverse events, partial remission, complete remission, objective response rate and other data; 4 . The control experiment includes the immune group and the chemotherapy group. The clinical trials that met the following criteria were excluded in this study: 1 . Early surgically resectable disease; 2. Research did not include overall survival rate, progression-free survival rate, incidence of adverse events, etc.; 3 . Patients had been treated with immunotherapy in advance ;4. Included less than 10 cases; 5 .Lacked effective data to evaluate the safety and effectiveness of immunotherapy; 6 . Repeated publications; 7 . The experiment did not include immunotherapy or chemotherapy; 8 . Violation of the above inclusion criteria

\section{End points}

The primary endpoint of the study was the overall survival (OS), defined as the time period between treatment initiation and death of any cause. Secondary endpoints included progression-free survival (PFS), objective response rate (ORR) and safety. PFS was defined as the time period between treatment initiation and the first documented disease progression or death of any cause, whereas the ORR, defined as the percentage of patients achieving a best response of complete response (CR) or partial response (PR).

\section{Statistical analysis}

Meta-analysis was performed using Review Manager, version 5.3 (RevMan; Cochrane Collaboration), a proprietary software provided by the Cochrane Collaboration. Hazard ratio (HR) and 95\% confidence interval (Cl) were the effect measures. Heterogeneity was determined by the $\chi^{2}$ test and $I^{2}$ test. If the heterogeneity was significant, the random effect model was adopted; otherwise, the fixed effect model was used. $p<0.05$ was considered statistically significant. $0<I^{2}<25 \%$, it means mild heterogeneity; $I^{2} \leq 50 \%$, it means moderate heterogeneity; $I^{2}>50 \%$, it means high heterogeneity. To explore the sources of heterogeneity, we performed a sensitivity analysis. The data statistical analysis was done by RevMan 5.3.

\section{Quality assessment}

The quality of included studies was assessed using the Cochrane Handbook 5.1.0 recommended risk of bias assessment tool[9], including: (1) random allocation method; (2) allocation concealment; (3) whether to adopt a blind method for the participants and researchers; (4) whether the outcome was assessed by a blind method; (5) completeness of outcome data; (6) selective reporting of outcomes; (7) other bias. The quality evaluation was 
conducted by two researchers independently, and in case of disagreement, a decision was made through discussion or by referring to the opinion of the third researcher.

\section{Result}

\section{Result of research}

According to the research strategy, 730 articles were searched first, and after deleting duplicate articles, 700 articles were deleted according to the title and abstract, and finally 30 articles were selected for comprehensive screening. After a comprehensive analysis of the articles, there were 2 articles lacking the necessary data, 2 articles were overlapping, and 22 articles lacked a suitable control group. In the end, 4 studies including 2087 patients were used for analysis. Table 1 presents the characteristics of the 4 included studies. The detailed research selection process is shown in the Figure.

(Fig.1)

Table 1 Characteristics of the included studies

\begin{tabular}{|c|c|c|c|c|c|c|}
\hline Clinical trial & Author & Year & NCT Number & $\mathrm{ICl}$ & CET & $\begin{array}{l}\text { Number } \\
\text { of } \\
\text { patients }\end{array}$ \\
\hline $\begin{array}{l}\text { ATTRACTION- } \\
3\end{array}$ & Kato K & 2019 & NCT02569242 & nivolumab & docetaxel/irinotecan & 419 \\
\hline ESCORT & $\begin{array}{l}\text { Huang } \\
\mathrm{J}\end{array}$ & 2020 & NCT03099382 & camrelizumab & paclitaxel/docetaxel & 448 \\
\hline $\begin{array}{l}\text { KEYNOTE- } \\
061\end{array}$ & $\begin{array}{l}\text { Shitara } \\
\text { K }\end{array}$ & 2018 & NCT02370498 & pembrolizumab & paclitaxel & 592 \\
\hline $\begin{array}{l}\text { KEYNOTE- } \\
181\end{array}$ & $\underset{T}{\text { Kojima }}$ & 2020 & NCT02559687 & pembrolizumab & paclitaxel/docetaxel/irinotecan & 628 \\
\hline
\end{tabular}

Abbreviations: ICI, Immune checkpoint inhibitor; CET, Chemical therapy; NCT, National clinical trial.

\section{Primary outcomes}

\section{Overall survival}

Four studies [10-13]directly compared the overall survival (OS) rate of patients after immunotherapy and chemotherapy, and the overall survival rate after immunotherapy was significantly improved (HR $0.77,95 \% \mathrm{Cl} 0.67-0.89, \mathrm{P}<0.001)$, the heterogeneity is moderate $\left(I^{2}=35 \%\right)$, and a random effects model is used(Fig.2a). And subgroup analysis showed that immunotherapy had a significant effect on the OS of esophageal squamous cell carcinoma (HR $0.75,95 \% \mathrm{Cl} 0.66-0.85$, $P<0.001)$ (Fig.2b), but the analysis results of esophageal adenocarcinoma showed no statistical significance $(P=0.02$, $\mathrm{I}^{2}=82 \%$ ) (Fig.2C). This indicates that in the treatment of patients with advanced esophageal cancer, immunotherapy significantly prolongs the survival time of patients compared with chemotherapy.

\section{Progression-free survival}


Progression-free survival (PFS) is also an important indicator for evaluating the safety of immunotherapy. Combining research [10-13] and analysis showed that there was no significant difference between the two groups $(\mathrm{HR} 1.01,95 \% \mathrm{Cl}$ $0.79-1.30)$. Due to the significant heterogeneity $\left(I^{2}=83 \%\right)$, a random effects model was used (Fig.3a). The results of subgroup analysis showed that immunotherapy had no difference in the progression-free survival of esophageal squamous cell carcinoma compared with chemotherapy (HR 0.88, 95\% Cl 0.68-1.14, P=0.33) (Fig.3b). Due to the lack of data on adenocarcinoma, the analysis of progression-free survival is temporarily not considered.

\section{Adverse events of grade 3 and above}

The incidence of adverse events after treatment is a major aspect of safety assessment. The analysis shows that compared with chemotherapy, immunotherapy has a significantly reduced incidence of grade 3 and above adverse events( HR 0.38; 95\% Cl 0.28-0.51), using random effects model $\left(\mathrm{I}^{2}=50 \%, P \otimes 0.001\right)$, belonging to moderate heterogeneity. Most of the adverse events such as Anemia, Asthenia, and Neutrophil count decreased (Fig.4a).

\section{Objective response rate}

Based on the analysis of four studies [10-13], the ORR of immunotherapy is more advantageous (HR $1.67 ; 95 \% \mathrm{Cl} 0.91-$ 3.06). Due to the high heterogeneity $\left({ }^{2}=78 \%\right)$, a random effects model is used (Fig.4b). Despite the high heterogeneity, immunotherapy has a longer sustained response to tumors and better anti-tumor properties than chemotherapy.

\section{Sensitivity analysis}

Re-examining the data and study selection did not reduce the heterogeneity. In order to ensure that the combined results are not subject to specific effects, we removed the included studies one by one for sensitivity analysis (Fig.5).

Obviously, the study by Kojima T and his colleagues [13]accounts for a large proportion. After excluding the study, the heterogeneity is negligible. Combining the data from the other three studies, (HR $0.72,95 \% \mathrm{Cl} 0.63-0.83 ; \mathrm{I}^{2}=0 \%$ ), which is also sufficient to prove the efficacy of immunotherapy. In the analysis of the incidence of adverse events of grade three and above, although the proportion of Kato $\mathrm{K}^{10}$ et al's research is not very large, it is still the most significant factor of heterogeneity. After excluding this study, the heterogeneity is reduced (HR $0.33,95 \% \mathrm{Cl} 0.26-0.42 ; \mathrm{I}^{2}=0 \%$ ), the results of the other three studies all prove the safety of immunotherapy relative to chemotherapy. For the progression-free survival rate, Huang J [10]'s heterogeneity contributed the most, and when the other 3 studies were analyzed $(\mathrm{HR} 1.14,95 \% \mathrm{Cl}$ $\left.1.02-1.28 ; I^{2}=0 \%\right)$, the median progression-free survival indicated chemotherapy is dominant, but the duration of remission is shorter than immunotherapy, showing that there is no significant difference between the two groups. Huang $\mathrm{J}[10]$ et al contributed to the heterogeneity of the objective response rate. After elimination, the heterogeneity decreased slightly, but still showed high heterogeneity $\left(1^{2}=61 \%\right)$, which may be due to the different drugs used in each study and the effects of drugs on tumors, because the efficacy of chemotherapy drugs is roughly the same. The response is also different, but immunotherapy still has advantages. In summary, the results still indicate the effectiveness and safety of immunotherapy relative to chemotherapy.

\section{Publication Bias test}

When analyzing the efficacy and safety of immunotherapy compared with chemotherapy in the second-line treatment of advanced esophageal cancer, since there are fewer studies involved and the heterogeneity is significantly reduced after excluding individual studies in the sensitivity analysis, the publication bias is not obvious.

\section{Discussion}


The above several studies have shown that after the first-line standard treatment for advanced or metastatic esophageal cancer, immunotherapy has shown excellent results compared with chemotherapy as the second-line treatment. In our meta-analysis, studies showed that the overall survival following immunotherapy in each study was significantly longer than that of chemotherapy, Although the effect on squamous cell carcinoma is far greater than adenocarcinoma, it still provides evidence for the effectiveness of immunotherapy in the treatment of esophageal cancer. For progression-free survival, there was no significant difference in the progression-free survival between the immunotherapy group and the chemotherapy group. Even the PFS of chemotherapy is longer than immunotherapy in most studies. In the ESCORT [10], although the median PFS of the two groups was similar (1.9 months), camrelizumab reduced the risk of disease progression/death by $31 \%$. The Kaplan-Meier survival curve reflects the long-lasting benefit of camrelizumab, and overall PFS is better than chemotherapy. The results of the ORIENT-2 study [14] announced at the ASCO annual meeting in 2020 , compared the effectiveness of sintilimab and chemotherapy in patients with esophageal squamous cell carcinoma after previous failure of first-line treatment. The results showed that the median OS in the sintilimab group was 7.2 months, and the median OS in the chemotherapy group was 6.2 months (HR 0.70, $P=0.032$ ). The 12-month OS rate in the sintilimab group was $37.4 \%$. The chemotherapy group was $21.4 \%$ but in the sintilimab vs chemotherapy group, the median PFS did not improve (1.6 months vs 2.9 months, HR 1.0, 95\% Cl 0.77-1.39, $P=0.989$ ). Compared with chemotherapy, sintilimab can improve the 6 -month PFS rate (20.6\% vs $15.7 \%)$, and the 12 -month PFS rate ( $10.4 \%$ vs $1.7 \%)$. This may indicate that the short-term effect of immune drugs in controlling disease progression is not as good as chemotherapy, but the long-term effect is better.

Regarding treatment-related adverse events, in our meta-analysis, among all the patients in the above study, the incidence of treatment-related adverse events was lower in the immunotherapy group than in the chemotherapy group, especially for adverse events of grade three and above. Studies have shown that the incidence within the immunotherapy group is smaller than that of chemotherapy group, which shows the tolerance of immunotherapy. Basically, these drugs have undergone preliminary clinical evaluations and have had preliminary experience in treatment. Common adverse events of grade 3 and above are diarrhea, anemia, fatigue, and neutropenia. This also provides further evidence for effective treatment of adverse events. Although the drug usage of the chemotherapy group in each trial is different, in the treatment of locally advanced esophageal cancer, multiple chemotherapy drugs such as paclitaxel, cisplatin, fluorouracil monotherapy or combination therapy have little effect on OS and the severity of adverse reactions is roughly the same [15-17]. However, immunotherapy has far fewer treatment-related adverse events of grade 3 and above than chemotherapy, which is of great significance to the prognosis and treatment of patients. The ORR of the nivolumab group in ATTRACTION-3 was similar to that of the chemotherapy group, but the remission lasted longer. In other studies $[10,12,13]$, the tumor objective response rate of the immune group was more obvious than that of the chemotherapy group and the time of duration was longer. These data have increased confidence in the effectiveness and safety of immunotherapy in the second-line treatment of advanced esophageal cancer.

PD-L1 expression by Tumor Proportion Score (TPS) and Combined Positive Score (CPS), as predictors of anti-pd-1/pd-I1 therapy, have certain significance in evaluating tumor prognosis and treatment effect [18-20]. The KEYNOTE-181[13] included patients with esophageal adenocarcinoma and esophageal squamous cell carcinoma. The combined positive score of PD-L1 on tumor expression and immune cell infiltration was used to evaluate the effect of PD-L1 expression on the outcome of pembrolizumab treatment. However, in the study of patients with esophageal squamous cell carcinoma, there is no comparative data showing that the combined positive score can enhance the efficacy more than tumor PD-L1 expression. ATTRACTION-3 showed that the survival benefit of nivolumab was not related to tumor PD-L1 expression, although patients with PD-L1 expression of at least $1 \%$ had a $15 \%$ higher risk of death than patients with PD-L1 expression below 1\%. In ESCORT, camrelizumab has clinical benefit in all PD-L1 expression subgroups, and patients with high PD-L1 expression seem to benefit more than patients with low PD-L1 expression. This result is similar to that observed in the attract-3 study, suggesting that the expression of PD-L1 may not be a reliable biomarker for predicting 
the outcome of esophageal squamous cell carcinoma. In a study [21], it was mentioned that the drug effect in the treatment of esophageal cancer is related to the long-term clinical response, but not to the expression of PD-L1. The relationship between the status of PD-L1 and the clinic has yet to be determined $[10,13,21]$.

One limitation of our analysis is that there are too few clinical trial data to perform subgroup analysis based on ICI types, and there is no evidence that different ICls have different effects on the efficacy and safety of neoadjuvant immunotherapy. Therefore, there is currently no first choice $\mathrm{ICl}$ in immunotherapy. And we have too little data on esophageal adenocarcinoma to conduct a comprehensive analysis. The choice of treatment should also be based on personal circumstances and clinical environment. Of course, follow-up research data is needed to prove this. In addition, although the OS, PFS, ORR and other indicators we studied and analyzed can initially confirm the effectiveness and safety of immunotherapy, the relatively small number of clinical trials included and the lack of data may lead to bias.

All in all, our meta-analysis proves the safety and effectiveness of immunotherapy for advanced esophageal cancer and provides support for its wide clinical application. However, the number of clinical trials is small, so more trials and studies are needed to verify it.

\section{Declarations}

Ethics approval and consent to participate: Not applicable.

Consent for publication: Not applicable.

Availability of data and materials: The datasets used and/or analysed during the current study are available from the corresponding author on reasonable request.

Competing interests: The authors declare that they have no competing interests.

Funding: This research did not receive any specific grant from funding agencies in the public, commercial, or not-forprofit sectors.

Authors' contributions: Kang-hao Zhu, Jian-fei Shen and Bao-fu Chen conceived and designed this study; Kang-hao Zhu and Dong Chen searched and collected the data; Kang-hao Zhu and Zi-xian Jin performed the statistical analysis and interpretation of data; Kang-hao Zhu, Cong-cong Xu and Si-jia Ren wrote the manuscript. All authors read and approved the final manuscript.

Acknowledgements: The authors wish to thank all the investigators. The authors wish to thank Taizhou Hospital of Zhejiang Province for providing the research environment.

\section{References}

1. Sung H, Ferlay J, Siegel RL, et al (2021) Global cancer statistics 2020: GLOBOCAN estimates of incidence and mortality worldwide for 36 cancers in 185 countries. CA Cancer J Clin. Feb 4.

2. Abnet CC, Arnold M, Wei WQ (2018) Epidemiology of Esophageal Squamous Cell Carcinoma. Gastroenterology. Jan;154(2):360-373.

3. Zhang HZ, Jin GF, Shen HB (2012) Epidemiologic differences in esophageal cancer between Asian and Western populations. Chin J Cancer. Jun;31(6):281-6.

4. Ajani JA, D'Amico TA, Bentrem DJ, et al (2019) Esophageal and Esophagogastric Junction Cancers, Version 2.2019, NCCN Clinical Practice Guidelines in Oncology. J Natl Compr Canc Netw. Jul 1;17(7):855-883.

Page $7 / 12$ 
5. Yang YM, Hong P, Xu WW, et al (2020) Advances in targeted therapy for esophageal cancer. Signal Transduct Target Ther. Oct 7;5(1):229.

6. Hong Y, Ding ZY (2019) PD-1 Inhibitors in the Advanced Esophageal Cancer. Front Pharmacol. Nov 29; $10: 1418$.

7. Xiao X, Hong HG, Zeng X, et al (2020) The Efficacy of Neoadjuvant Versus Adjuvant Therapy for Resectable Esophageal Cancer Patients: A Systematic Review and Meta-Analysis. World J Surg. Dec;44(12):4161-4174.

8. McKenzie JE, Bossuyt PM, Boutron I, et al (2021) The PRISMA 2020 statement: an updated guideline for reporting systematic reviews. BMJ;372: $\mathrm{n} 71$.

9. Higgins JP, Altman DG, Gøtzsche PC, et al (2011) The Cochrane Collaboration's tool for assessing risk of bias in randomised trials. BMJ. Oct 18;343: d5928.

10. Huang J, Xu J, Chen Y, et al (2020) ESCORT Study Group. Camrelizumab versus investigator's choice of chemotherapy as second-line therapy for advanced or metastatic oesophageal squamous cell carcinoma (ESCORT): a multicentre, randomised, open-label, phase 3 study. Lancet Oncol. Jun;21(6):832-842.

11. Kato K, Cho BC, Takahashi M, et al (2019) Nivolumab versus chemotherapy in patients with advanced oesophageal squamous cell carcinoma refractory or intolerant to previous chemotherapy (ATTRACTION-3): a multicentre, randomised, open-label, phase 3 trial. Lancet Oncol. Nov;20(11):1506-1517.

12. Shitara K, Özgüroğlu M, Bang YJ, et al (2018) KEYNOTE-061 investigators. Pembrolizumab versus paclitaxel for previously treated, advanced gastric or gastro-oesophageal junction cancer (KEYNOTE-061): a randomised, openlabel, controlled, phase 3 trial. Lancet. Jul 14;392(10142):123-133.

13. Kojima T, Shah MA, Muro K, Francois E, et al (2020) KEYNOTE-181 Investigators. Randomized Phase III KEYNOTE181 Study of Pembrolizumab Versus Chemotherapy in Advanced Esophageal Cancer. J Clin Oncol. Dec 10;38(35):4138-4148.

14. Xu JM, Fan QX, Wu ZJ, et al (2020) Sintilimab in patients with advanced esophageal squamous cell carcinoma refractory to previous chemotherapy: A randomized, open-label phase II trial (ORIENT-2) [J]. J Clin Oncol 38: (suppl; abstr 4511).

15. Mizota A, Shitara K, Kondo C, et al (2011) A retrospective comparison of docetaxel and paclitaxel for patients with advanced or recurrent esophageal cancer who previously received platinum-based chemotherapy. Oncology;81(34):237-42.

16. Shirakawa T, Kato K, Nagashima K, et al (2014) A retrospective study of docetaxel orpaclitaxel in patients with advanced or recurrent esophageal squamous cell carcinoma who previously received fluoropyrimidine- and platinum-based chemotherapy. Cancer Chemother Pharmacol;74(6):1207-15.

17. Lordick F, von Schilling C, Bernhard H, et al (2003), Peschel C. Phase II trial of irinotecan plus docetaxel in cisplatinpretreated relapsed or refractory oesophageal cancer. Br J Cancer;89(4):630-3.

18. Azuma K, Ota K, Kawahara A, et al (2014) Association of PD-L1 overexpression with activating EGFR mutations in surgically resected nonsmall-cell lung cancer. Ann Oncol;25(10):1935-1940.

19. Okita R, Maeda A, Shimizu K, et al (2017). PD-L1 overexpression is partially regulated by EGFR/HER2 signaling and associated with poor prognosis in patients with non-small-cell lung cancer. Cancer Immunol Immunother;66(7):865-876.

20. Wu P, Wu D, Li L, Chai Y, Huang J (2015) PD-L1 and Survival in Solid Tumors: A Meta-Analysis. PLoS One;10(6): e0131403.

21. Xu J, Bai Y, Xu N, et al (2020) Tislelizumab Plus Chemotherapy as First-line Treatment for Advanced Esophageal Squamous Cell Carcinoma and Gastric/Gastroesophageal Junction Adenocarcinoma. Clin Cancer Res;26(17):4542-4550. 


\section{Figures}

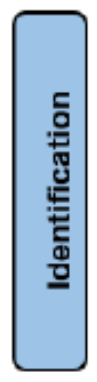

725 of records identified through database scarching

5 of additional records identified

through other sourees

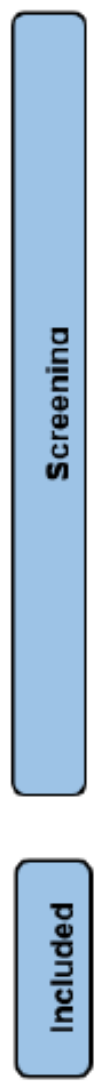

700 of records excluded
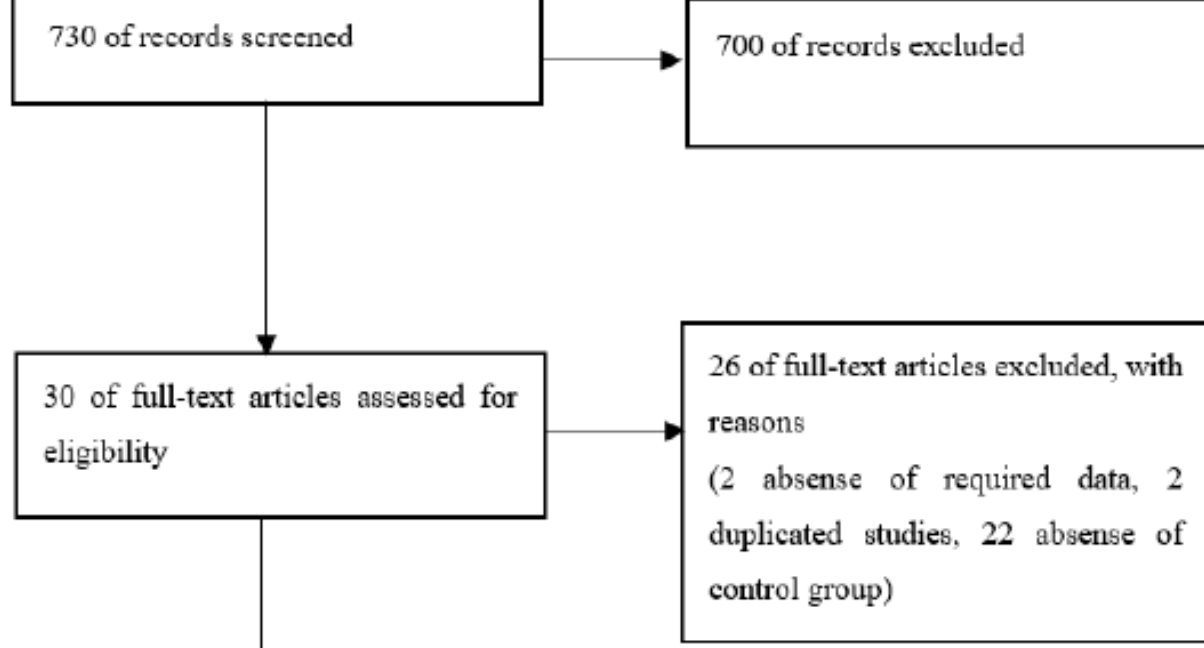

\section{Figure 1}

Publication search. Publications were initially identified through PubMed, Embase, and Cochrane library searches, which used the terms esophageal cancer (including esophageal squamous cell carcinoma, esophageal adenocarcinoma), programmed cell death protein-1, and immunotherapy (including all currently known immune checkpoints inhibitors). After eliminating the duplicate reference, through the review of titles and abstracts, the selected 730 publications were screened, and initially 30 suitable publications were obtained. The contents of these 30 references were thoroughly and carefully reviewed, and 4 studies were finally included for qualitative analysis. 
Hazard Ratio

Studv or Subgroup log[Hazard Ratio]
ATTRACTION-3

ESCORT

KEYNOTE-061

KEYNOTE-181

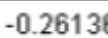

$-0.34249$

$\begin{array}{ll}-0.4943 & 0.20057\end{array}$

$\begin{array}{ll}-0.11653 & 0.085835\end{array}$

Total $(95 \% \mathrm{Cl})$

Test for overall effect: $Z=3.59(P=0.0003)$
$100.0 \%$

E Weight IV, Random, 95\% Cl

$0.77[0.62,0.96]$

$0.71[0.57,0.88]$

$0.61[0.41,0.90]$

$0.89[0.75,1.05]$

$0.77[0.67,0.89]$

b

Odds Ratio

SE Weight IN, Random, $95 \% \mathrm{Cl}$

Study or Subgroup log[Odds Ratio] ATTRACTION-3

ESCORT

KEYNOTE-181

Total $(95 \% \mathrm{Cl})$

Heterogeneity. $\mathrm{Tau}^{2}=0.00 ; \mathrm{Ch}^{2}=0.37, \mathrm{df}=2(\mathrm{P}=0.83) ; \mathrm{I}^{2}=0 \%$

Test for overall effect: $Z=4.60(P<0.00001)$
$0.77[0.62,0.96]$

$0.71[0.57,0.88]$

$0.77[0.62,0.95]$

$0.75[0.66,0.85]$

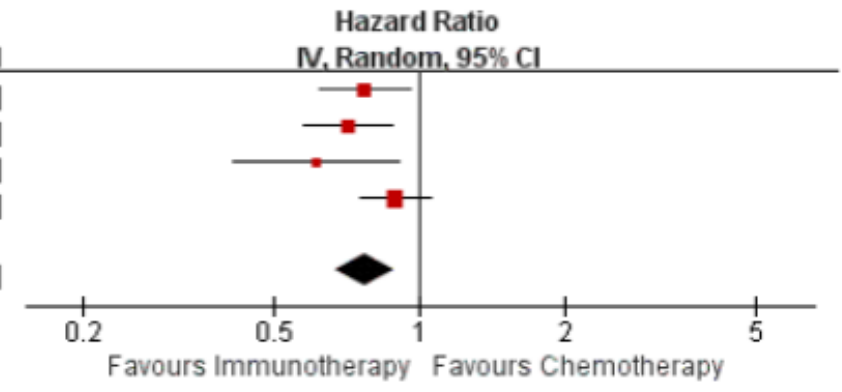

Favours Immunotherapy Favours Chemotherapy

Hazard Ratio

, Random, 95\% C

c

Odds Ratio Odds Ratio

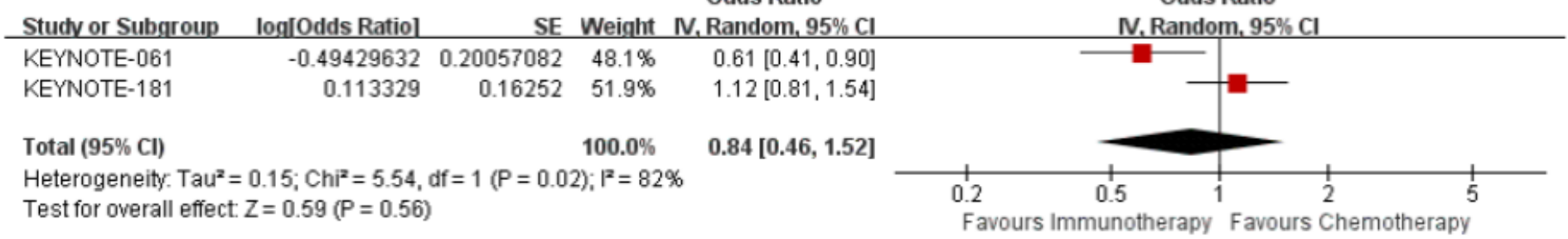

\section{Figure 2}

Comparison of forest plot in OS: Immunotherapy vs Chemotherapy. (a) all patients. (b) Esophageal squamous cell carcinoma Subgroup. (c) Esophageal adenocarcinoma subgroup. 


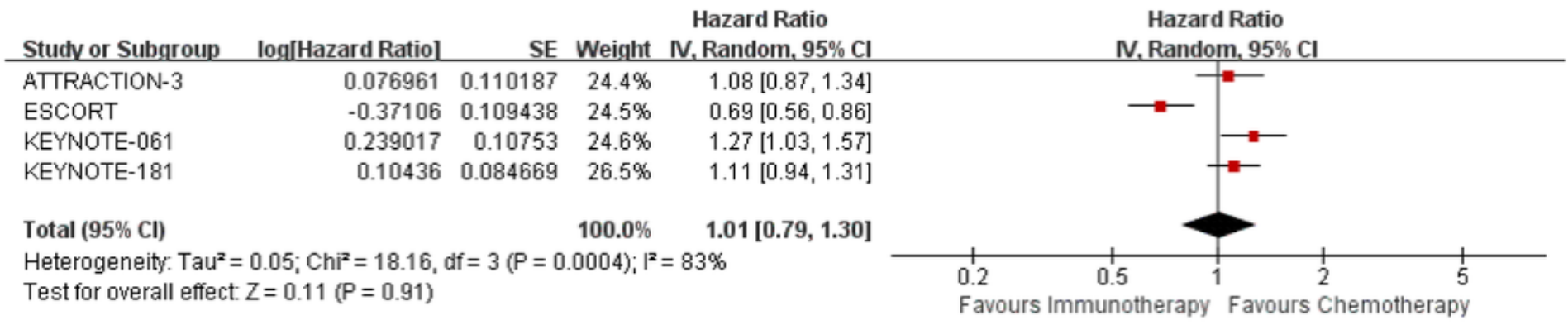

b

Odds Ratio

Odds Ratio

Study or Subgroup log[Odds Ratio]

SE Weight IV, Random, $95 \% \mathrm{Cl}$

N. Random, 95\% C

ATTRACTION-3

$\begin{array}{lll}0.076961 & 0.11018665 & 33.0 \%\end{array}$

$1.08[0.87,1.34]$

ESCORT

$\begin{array}{lll}-0.37106368 & 0.10943765 & 33.1 \%\end{array}$

$0.69[0.56,0.86]$

KEYNOTE-181

$\begin{array}{lll}-0.08338161 & 0.10456625 & 33.8 \%\end{array}$

$0.92[0.75,1.13]$

Total $(95 \% \mathrm{Cl})$

$100.0 \%$

Heterogeneity: $\mathrm{Tau}^{2}=0.04 ; \mathrm{Chi}^{2}=8.57, \mathrm{df}=2(\mathrm{P}=0.01) ; \mathrm{I}^{2}=77 \%$

Test for overall effect: $Z=0.97(P=0.33)$

$0.88[0.68,1.14]$

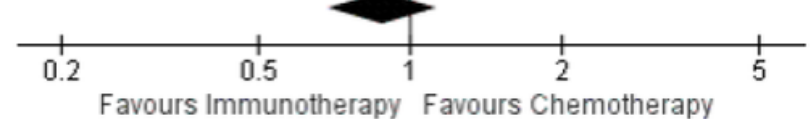

\section{Figure 3}

Comparison of forest plot in PFS: Immunotherapy vs Chemotherapy. (a) all patients. (b) Esophageal squamous cell carcinoma Subgroup.

a

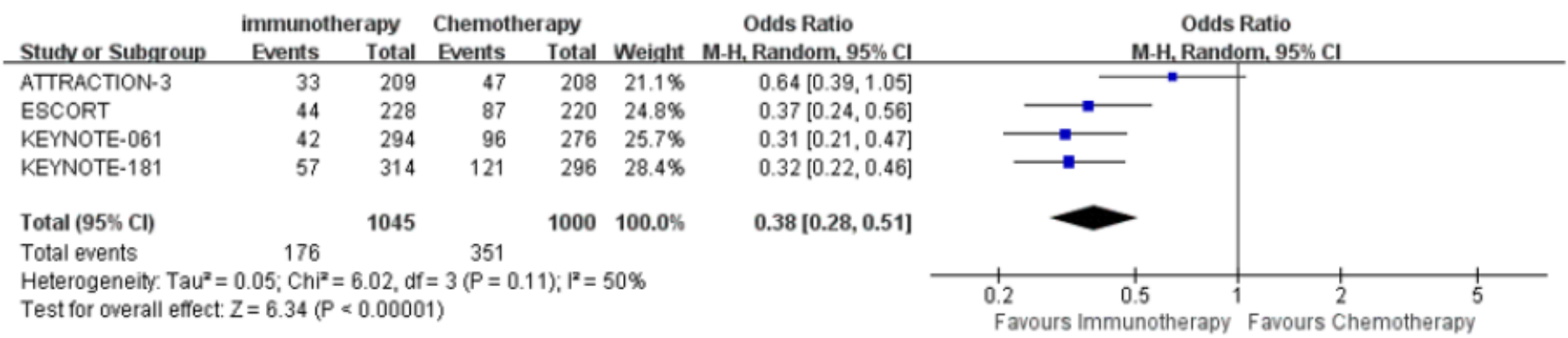

b

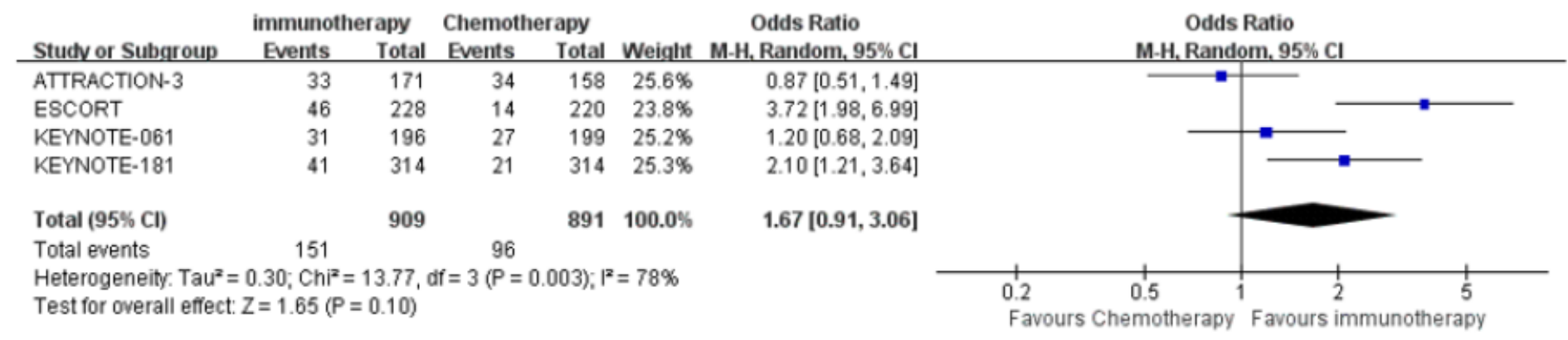


Figure 4

Comparison of forest plot: Immunotherapy vs Chemotherapy. (a) Adverse events of grade 3 and above. (b) Objective response rate.

a

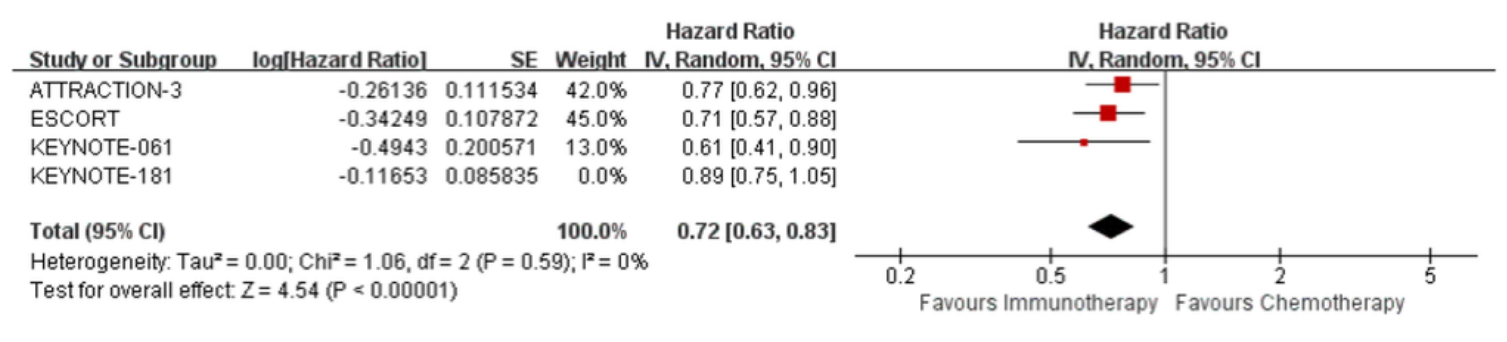

b

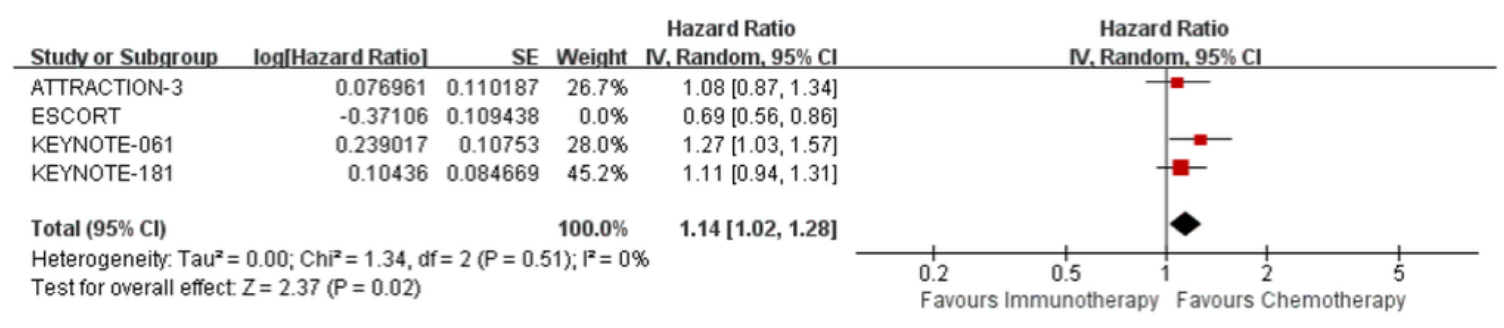

c

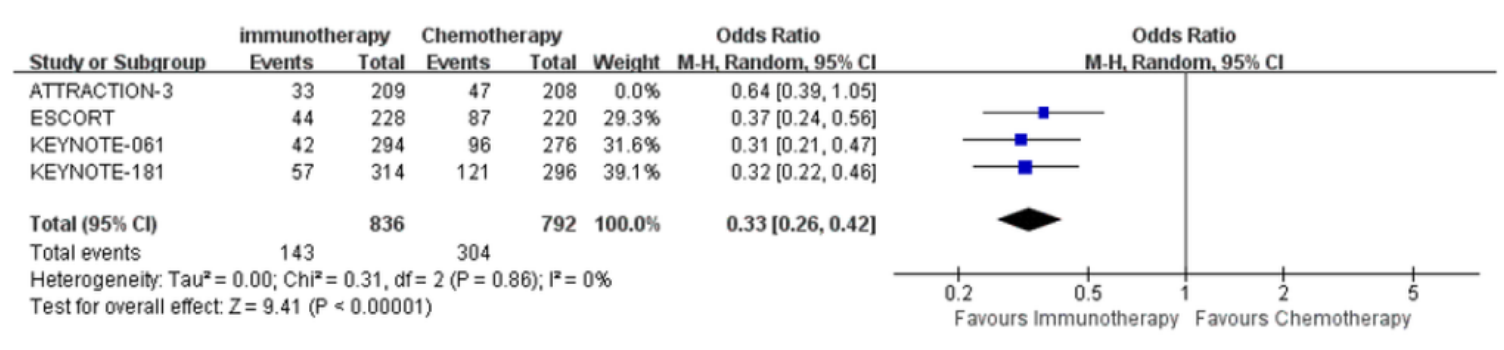

$\mathrm{d}$

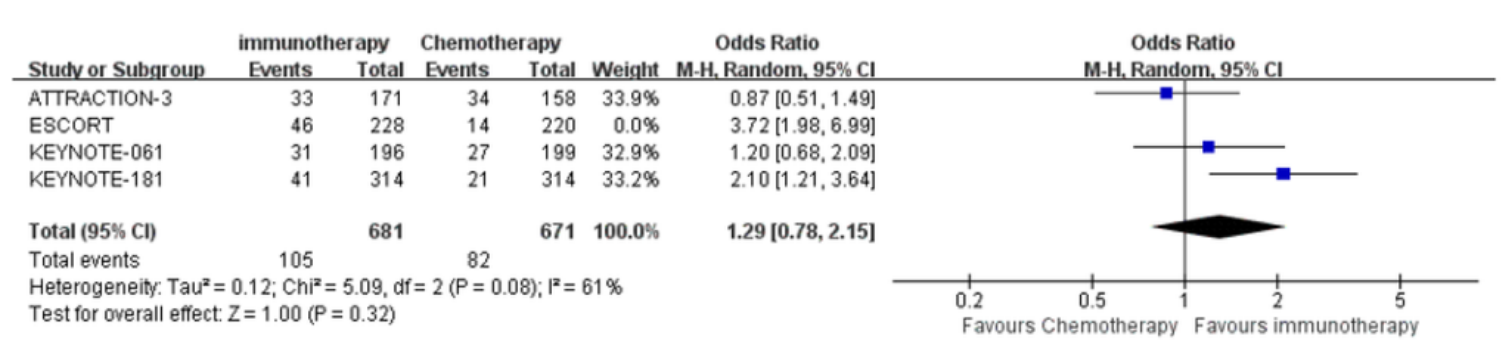

\section{Figure 5}

Comparison of forest plot in sensitivity analysis: Immunotherapy vs Chemotherapy. (a) Overall survival. (b) Progressionfree survival. (c) Adverse events of grade 3 and above. (d) Objective response rate 